\title{
A clinical scoring system to identify patients with sebaceous neoplasms at risk for the Muir-Torre variant of Lynch syndrome
}

\author{
Maegan E. Roberts, MS', Douglas L. Riegert-Johnson, MDD,3, Brittany C. Thomas, MS ${ }^{4}$, \\ Kandelaria M. Rumilla, MD ${ }^{4}$, Colleen S. Thomas, MS5, Michael G. Heckman, MS ${ }^{5}$, Jennifer U. Purcell, BS $^{6}$, \\ Nancy B. Hanson, MS', Kathleen A. Leppig, MD', Justin Lim, BS ${ }^{7}$ and Mark A. Cappel, MD ${ }^{8}$
}

\begin{abstract}
Purpose: The Muir-Torre syndrome variant of Lynch syndrome is characterized by the presence of sebaceous neoplasms (adenoma, epithelioma/sebaceoma, carcinoma) and Lynch syndrome-associated cancers (colon, endometrial, and others). Several clinical scoring systems have been developed to identify patients with colon cancer at high risk of Lynch syndrome. However, no such system has been described for patients presenting with sebaceous neoplasms.

Methods: Based on logistic regression analysis, a scoring system was developed for patients with sebaceous neoplasm to identify those with the highest likelihood of having Muir-Torre syndrome. The final version of the scoring system included variables such as age at presentation of initial sebaceous neoplasm, total number of sebaceous neoplasms, personal history of a Lynch-related cancer, and family history of Lynch-related cancers.
\end{abstract}

Results: Patients with a score of 3 or more were more likely to have Muir-Torre syndrome ( 28 of 29 patients), those with a score of 2 had intermediate likelihood (12 of 20 patients), and no patient with a score of 0 or 1 was diagnosed with Muir-Torre syndrome.

Conclusion: The Mayo Muir-Torre syndrome risk scoring system appears to identify whether patients who present with sebaceous neoplasms are in need of further Lynch syndrome evaluation using easily ascertained clinical information. Abnormal mismatch repair gene immunohistochemistry of a sebaceous neoplasm is a poor predictor in regard to diagnosing Lynch syndrome.

Genet Med advance online publication 6 March 2014

Key Words: immunohistochemistry; mismatch repair genes; Muir-Torre syndrome; sebaceous adenoma; sebaceous neoplasms

\section{INTRODUCTION}

The Muir-Torre syndrome (MTS) variant of Lynch syndrome (LS) is characterized by the presence of sebaceous neoplasms (SNs; adenoma, epithelioma/sebaceoma, carcinoma) and LS-associated cancers (colon, endometrial, urothelial, and others). MTS and LS are caused by mutations in or, in rare cases, hypermethylation of the DNA mismatch repair (MMR) genes. MMR immunohistochemistry (IHC) is done on colon cancer tissue to screen for LS. MMR IHC is also routinely requested for SNs; however, the clinical utility of this test is still unclear. Although many groups have reported the results of MMR IHC on SNs, very few have correlated this with germline genetic testing of MMR genes. Previously, we reported on a group of 90 patients who had at least one pathologically confirmed SN. ${ }^{1}$ MMR IHC had much lower sensitivity and specificity for LS compared with MMR IHC done on colon tumors ( $85 \%$ vs. $92-94 \%$ and $48 \%$ vs. $88-100 \%$, respectively)..$^{2-4}$ The specificity of MMR IHC on colon tumors varies depending on whether the loss of MLH1 and PMS2 due to epigenetic silencing (sporadic hypermethylation) of the $h M L H 1$ promoter is taken into consideration. It has been estimated that $10-15 \%$ of colon cancers will be sporadic but will show loss of MLH1 and PMS2 when MMR IHC is done, due to $h M L H 1$ promoter hypermethylation. ${ }^{5} h M L H 1$ hypermethylation could be a partial, but not complete, explanation for the lack of performance of MMR IHC on SNs, as loss of MSH2 and MSH6 has also been shown to be unreliable. We have not been able to find MMR gene hypermethylation data on a large number of SNs.

To a practicing clinician, the knowledge of the pretest probability of identifying a germline gene mutation is crucial in advising patients whether to proceed with genetic testing and bear the associated financial and psychosocial burdens. The conclusions that could be drawn from our previous work in this regard were limited because only $16 \%$ of patients had germline MMR genetic testing done. This study addresses the above limitation by evaluating a large number of SN patients who had both MMR IHC and germline MMR genetic tests completed. The study also examines whether MMR IHC is useful to screen SN patients for

${ }^{1}$ Genetic Counseling, GeneDx, Gaithersburg, Maryland, USA; ${ }^{2}$ Department of Medical Genetics, Mayo Clinic, Jacksonville, Florida, USA; ${ }^{3}$ Department of Gastroenterology, Mayo Clinic, Jacksonville, Florida, USA; ${ }^{4}$ Laboratory Medicine and Pathology, Mayo Clinic, Rochester, Minnesota, USA; ${ }^{5}$ Department of Health Sciences Research, Mayo Clinic, Jacksonville, Florida, USA; ${ }^{6}$ Clinical Research Internship Study Program, Mayo Clinic, Jacksonville, Florida, USA; ${ }^{7}$ Department of Genetics, Group Health Cooperative, Seattle, Washington, USA; ${ }^{8}$ Department of Dermatology, Mayo Clinic, Jacksonville, Florida, USA. Correspondence: Maegan E. Roberts (mroberts@genedx.com) 


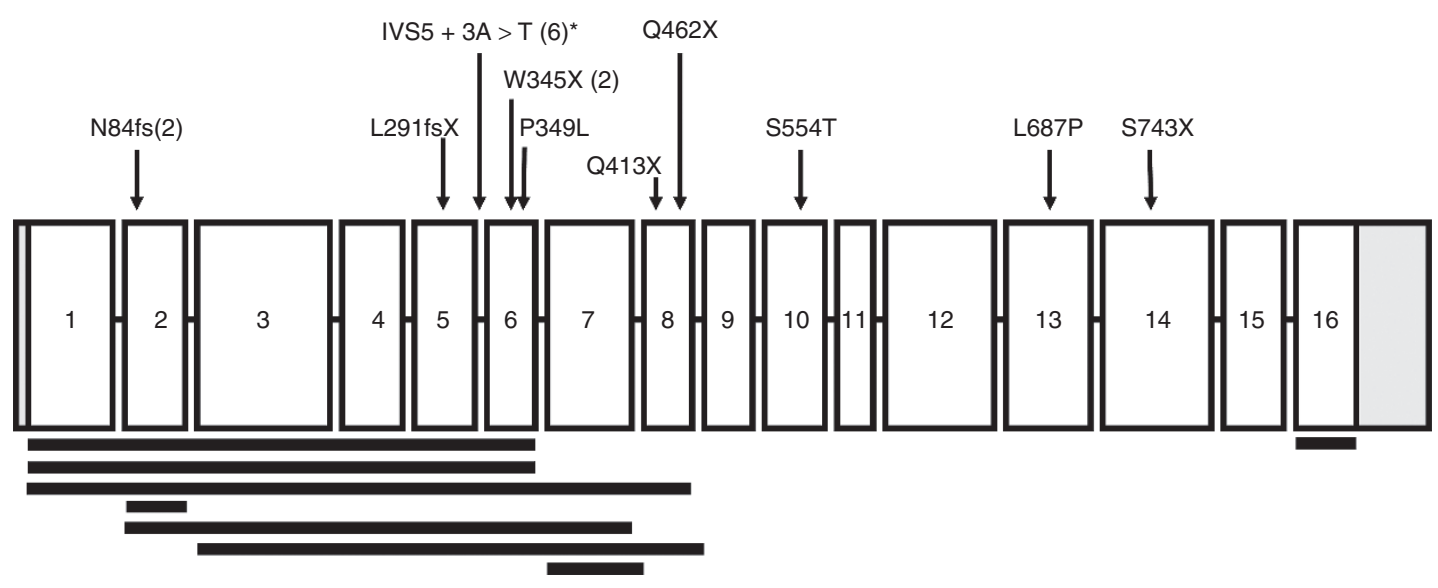

Figure 1 hMSH2 mutations in Muir-Torre Lynch syndrome patients. Point mutations shown above with deletions shown below. Mutations in $h M S H 2$ most commonly involved exons 5 or $6(60 \%)$, and there was an overrepresentation of the common splice site mutation IVS5+3A>T (24\% of $h M S H 2$ mutations). In our laboratory, the IVS5+3A>T mutation makes up 10\% of all identified deleterious hMSH2 mutations. Therefore, it appears that this mutation is overrepresented in our Muir-Torre patient cohort and may be associated with risk for the MTS phenotype. The IVS5+3A>T mutation disrupts the intron 5 donor splice site, resulting in transcripts lacking exon $5 .^{7}$

germline genetic testing (as it is used for patients with colon cancer).

\section{METHODS}

\section{Definitions and approval}

Lynch-related cancers include colorectal, endometrial, ovarian, small bowel, urinary tract (renal pelvis and ureter), biliary tract, and others. A patient was suspected to have MTS if he/she had a history of at least one SN (carcinoma, epithelioma/sebaceoma, or adenoma) and if germline genetic testing showed a pathogenic mutation in any one of the known MMR genes ( $h M L H 1$, $h M S H 2, h M S H 6, P M S 2$, or EPCAM). The study was approved by the Mayo Clinic Institutional Review Board.

\section{Subjects}

Cancer-specific genetic counselors were identified via the National Society of Genetic Counselors website (http://www. nsgc.org). All 511 genetic counselors who listed "cancer" as their area of practice were sent an e-mail requesting them to complete a Web-based REDCap survey regarding their experience with SN patients referred to them for concerns of MTS as a possible diagnosis. Of the 511 genetic counselors contacted, 53 (10\%) responded. Data collected include geographic region, years of experience, work setting, number of patients referred to evaluate for MTS based on the presence of SNs in the past 5 years, experience with MMR IHC of SNs, and experience with germline MMR genetic testing in patients with a history of at least one SN. A descriptive summary of the 53 responding genetic counselors is presented in Supplementary Tables S1-S3 online. The genetic counselors provided information on a total of 149 patients. Patients were excluded from the analysis if germline MMR genetic testing was not completed $(n=57)$. Among 92 patients who had genetic testing completed, 2 had variants of uncertain significance, and 1 patient did not have the results available, resulting in 89 patients for the final analysis.
A descriptive summary of the characteristics of the 89 patients included in the analysis and the 60 patients excluded is shown in Supplementary Table S4 online.

\section{Statistical analysis}

Among the patients who had at least one abnormal MMR IHC finding of a SN, the proportion of patients who did not have MTS based on germline genetic testing was estimated along with an exact binomial 95\% confidence interval (CI). The associations between patient characteristics and diagnosis of MTS were evaluated using logistic regression models in which odds ratios and CIs were estimated and in which only risk factors occurring in at least five patients were considered. In order to adjust for the number of statistical tests performed in our logistic regression analysis, we employed a Bonferroni correction, after which $P$-values of 0.003 or less were considered statistically significant. Based on the results of logistic regression analysis, a scoring algorithm was developed to identify patients with the highest likelihood of MTS. Area under the receiver operating characteristic curve, sensitivity, specificity, positive predictive value, and negative predictive value were estimated along with 95\% CIs. Positive and negative predictive values were estimated under the assumption that the percentage of patients with MTS in the population of patients with genetic testing for MTS done is similar to the percentage of patients with MTS observed in our data. All analyses were performed using SAS (version 9.3; SAS Institute, Cary, NC).

\section{RESULTS}

\section{Patient characteristics}

Patient characteristics are summarized in Supplementary Table S5 online. Of the 89 patients included in the study, $40(45 \%)$ had MTS based on their genetic testing showing a germline MMR gene mutation. Sixty-six (74\%) patients were men, with a median age at presentation of first SN of 56 years 
Table 1 Associations with Muir-Torre syndrome

\begin{tabular}{|c|c|c|c|}
\hline \multirow[b]{2}{*}{ Variable } & \multirow{2}{*}{$\begin{array}{c}\text { Fraction (\%) } \\
\text { with MTS }\end{array}$} & \multicolumn{2}{|c|}{ Association with MTS } \\
\hline & & OR $(95 \% \mathrm{Cl})$ & $P$-value \\
\hline \multicolumn{4}{|l|}{ Gender } \\
\hline Female & $11 / 23(48 \%)$ & 1.00 (reference) & 0.75 \\
\hline Male & 29/66 (44\%) & $0.86(0.33-2.22)$ & \\
\hline \multicolumn{4}{|c|}{ History of immunosuppressant drugs } \\
\hline No & $39 / 83(47 \%)$ & 1.00 (reference) & 0.18 \\
\hline Yes & $1 / 6(17 \%)$ & $0.23(0.03-2.02)$ & \\
\hline \multicolumn{4}{|c|}{ Age at presentation of first $\mathrm{SN}$} \\
\hline $\begin{array}{l}60 \text { years or } \\
\text { older }\end{array}$ & $9 / 36(25 \%)$ & 1.00 (reference) & 0.002 \\
\hline $\begin{array}{l}\text { Younger than } \\
60 \text { years }\end{array}$ & $31 / 53(58 \%)$ & $4.23(1.67-10.73)$ & \\
\hline \multicolumn{4}{|c|}{ Total number of SNs } \\
\hline 1 & $19 / 66(29 \%)$ & 1.00 (reference) & $<0.001$ \\
\hline 2 or more & $21 / 22(95 \%)$ & $51.95(6.52-414.00)$ & \\
\hline \multicolumn{4}{|c|}{ Personal history of any Lynch-related cancer } \\
\hline No & $17 / 62(27 \%)$ & 1.00 (reference) & $<0.001$ \\
\hline Yes & $23 / 27(85 \%)$ & $15.22(4.59-50.48)$ & \\
\hline
\end{tabular}

Personal history of colon cancer

$\begin{array}{lccc}\text { No } & 23 / 71(32 \%) & 1.00(\text { reference }) & <0.001 \\ \text { Yes } & 17 / 18(94 \%) & 35.48(4.45-283.17) & \end{array}$

Personal history of endometrial cancer

$\begin{array}{lrlr}\text { No } & 7 / 17(41 \%) & 1.00 \text { (reference) } & 0.29 \\ \text { Yes } & 4 / 6(67 \%) & 2.86(0.41-20.14) & \end{array}$

Personal history of urinary tract cancer ${ }^{\mathrm{a}}$

No $\quad 35 / 84(42 \%) \quad 1.00$ (reference) $\quad 0.068$

Yes $\quad 5 / 5(100 \%) \quad 15.34(0.82-286.35)$

Family history of any Lynch-related cancer

$\begin{array}{lrll}\text { No } & 4 / 29(14 \%) & 1.00 \text { (reference) } & <0.001 \\ \text { Yes } & 36 / 60(60 \%) & 9.38(2.90-30.36)\end{array}$

Family history of colon cancer

$\begin{array}{llll}\text { No } & 7 / 40(18 \%) & 1.00 \text { (reference) } & <0.001 \\ \text { Yes } & 33 / 49(67 \%) & 9.72(3.54-26.71) & \end{array}$

Family history of endometrial cancer

$\begin{array}{lll}\text { No } \quad 29 / 74(39 \%) & 1.00 \text { (reference) } & 0.021\end{array}$

Yes $\quad 11 / 15(73 \%) \quad 4.27(1.24-14.69)$

Family history of urinary tract cancer

$\begin{array}{lrll}\text { No } & 31 / 78(40 \%) & 1.00 \text { (reference) } & 0.019 \\ \text { Yes } & 9 / 11(82 \%) & 6.82(1.38-33.72) & \end{array}$

Amsterdam II criteria

$\begin{array}{llcl}\text { No } & 22 / 70(31 \%) & 1.00 \text { (reference) } & <0.001 \\ \text { Yes } & 18 / 19(95 \%) & 39.27(4.93-313.08) & \end{array}$

Odds ratios (ORs) and $95 \%$ confidence intervals ( $\mathrm{Cls}$ ) were estimated from single-variable logistic regression models.

MTS, Muir-Torre syndrome; SN, sebaceous neoplasm (sebaceous adenoma, epithelioma/sebaceoma, and carcinoma).

aDue to the fact that all patients with a personal history of urinary tract cancer had MTS, logistic regression was not possible, and a value of 0.5 was added to each cell to estimate the $\mathrm{OR}, \mathrm{Cl}$, and $P$ value. (range, 30-88 years), 22 (25\%) had more than 1 SN, 27 (30\%) had a personal history of a Lynch-related cancer, and 60 (67\%) had a family history of Lynch-related cancers. Seventy-four (83\%) patients had MMR IHC results recorded on at least one SN, three of which had normal MMR IHC on all SNs tested, and 71 had abnormal MMR IHC results on at least one SN. Two of these patients had discordant results, both with normal MMR IHC reported on one SN and abnormal MMR IHC reported on another.

For the 40 patients identified as harboring a germline MMR gene mutation, 27 (68\%) had mutations in the $h M S H 2$ gene, 9 $(23 \%)$ in the $h M L H 1$ gene, $4(10 \%)$ in the $h M S H 6$ gene, and no mutations in the PMS2 gene were identified. There were 17 MTS patients (MMR mutation positive) who had a history of at least one SN and a personal history of a colorectal cancer (CRC). Interestingly, 3 had developed at least one $\mathrm{SN}$ prior to their CRC; 12 of the 17 patients developed CRC before they developed their first $\mathrm{SN}$, and 2 presented with synchronous CRC and SN. In the 12 patients who developed CRC prior to their first $\mathrm{SN}$, the median time between the development of $\mathrm{CRC}$ and $\mathrm{SN}$ was 7 years (range, 1-34 years). The data for the observed MMR IHC staining patterns were available for 34 of our 40 MTS patients. Of these 34 patients, 7 (21\%) had at least one SN with a MMR IHC staining pattern that was not consistent with the germline MMR gene mutations identified.

\section{Possible genotype-phenotype correlation}

hMSH2 mutation carriers were overrepresented (68\% of cases), followed by $h M L H 1$ (23\%) and hMSH6 (10\%); no PMS2 mutation carriers were identified (Figure 1 outlines the identified $h M S H 2$ mutations). Of 27 patients identified as having a germline $h \mathrm{MSH} 2$ mutation, exact $h \mathrm{MSH} 2$ mutations were reported in 25 patients (93\%). Mutations in hMSH2 most commonly involved exons 5 or $6(60 \%)$. The IVS5+3A $>$ T $h M S H 2$ mutation was overrepresented as compared to the experience of the Mayo Clinic DNA diagnostic laboratory (24\% of MTS $h M S H 2$ mutations vs. $10 \%$ of all identified deleterious $h M S H 2$ mutations). This suggests that the IVS5 $+3 \mathrm{~A}>\mathrm{T}$ genotype may be associated with an increased risk for the MTS phenotype.

\section{Estimated false-positive rate of MMR IHC on SNs}

Among the 71 patients who had abnormal MMR IHC of one or more SNs, 40 (56\%; 95\% CI: 44-68\%) showed no germline MMR mutation after genetic testing. This supports our previous finding that MMR IHC of SNs is prone to false-positive results.

\section{Evaluation of associations with MTS}

The associations of patient characteristics with MTS based on germline MMR gene mutation testing are summarized in Table 1. In single-variable logistic regression analysis, the factors significantly associated $(P<0.003)$ with MTS after multiple testing adjustment included age at presentation of first $\mathrm{SN}$ of less than 60 years, two or more SNs, personal history of any 
Table 2 The Mayo Muir-Torre syndrome risk score algorithm

\begin{tabular}{lc} 
Variable & Score \\
\hline Age at sebaceous neoplasma diagnosis (years) & \\
60 or older & 1 \\
Younger than 60 & \\
Total number of sebaceous neoplasms & 0 \\
1 & 2 \\
2 or more & \\
Personal history of any Lynch-related cancer & 0 \\
No & 1 \\
Yes & \\
Family history of any Lynch-related cancer & 0 \\
No & 1 \\
\hline Yes
\end{tabular}

Scores for the four variables are summed to create a total score, the "Mayo MTS risk score," with a possible range of $0-5$. A score of 2 or more has a sensitivity of $100 \%$ and specificity of $81 \%$ for predicting a germline mutation in a Lynch syndrome mismatch repair gene (Tables 3 and 4 ).

MTS, Muir-Torre syndrome.

aThe Mayo MTS risk score is applicable to patients with sebaceous adenomas, sebaceous epitheliomas/sebaceomas, and sebaceous carcinomas. Sebaceous hyperplasia is not a component of the Muir-Torre variant of Lynch syndrome, and the Mayo MTS risk score is not applicable to patients with only sebaceous hyperplasia.

bynch syndrome-related cancers include colorectal, endometrial, ovarian, small bowel, urinary tract (renal pelvis and ureter), and biliary tract cancers

Table 3 Proportion of patients with Muir-Torre syndrome according to Mayo Muir-Torre syndrome risk score

\begin{tabular}{lcc} 
& \multicolumn{2}{c}{ Proportion of patients with MTS } \\
\cline { 2 - 3 } Mayo MTS risk score & Fraction (\%) & $\begin{array}{c}95 \% \text { Confidence } \\
\text { interval }\end{array}$ \\
\hline 0 & $0 / 10(0 \%)$ & $0-31 \%$ \\
\hline 1 & $0 / 29(0 \%)$ & $0-12 \%$ \\
\hline 2 & $12 / 20(60 \%)$ & $36-81 \%$ \\
\hline 3 & $11 / 11(100 \%)$ & $72-100 \%$ \\
\hline 5 & $10 / 11(91 \%)$ & $59-100 \%$ \\
\hline
\end{tabular}

The Mayo Muir-Torre syndrome risk score was not calculated for one patient due to missing data (total number of sebaceous neoplasms).

MTS, Muir-Torre syndrome.
Lynch-related cancer or colon cancer, family history of any Lynch-related cancer or colon cancer, and Amsterdam II criteria pedigree. Multivariable analysis was not performed in view of the relatively small number of MTS patients and the high degree of correlation between many of factors evaluated for association with MTS.

\section{Development of a scoring system for risk of MTS}

To identify patients with the highest likelihood of MTS and who therefore should undergo germline MMR genetic testing, we developed a scoring algorithm from the results of logistic regression analysis. Of the seven factors significantly associated with MTS, we eliminated personal history and family history of colon cancer from consideration in our scoring algorithm and retained personal history and family history of Lynchrelated cancers (which includes colon cancer), to err on side of inclusion rather than exclusion. Both personal and family history of Lynch-related cancers were retained in the scoring system due to the degree of independence of these two measures; although only 2 of 27 patients with a personal history did not have a family history, 35 of 62 patients with no personal history did have a family history. We eliminated Amsterdam II criteria due to its high correlation with personal and family history of Lynch-related cancer and also due to its complexity. We utilized the four remaining variables (presentation of first SN prior to the age of 60 years, two or more SNs, personal history of a Lynch-related cancer, family history of Lynch-related cancers) in our scoring algorithm. We assigned each category of these characteristics an individual score and summed these individual scores to create a total score, called the "Mayo MTS risk score." For all risk factors, the absence of a given risk factor was assigned a score of 0 , and its presence was assigned a score of 1 , except two or more SNs, which, due to its extremely high odds ratio and individual predictive ability, was assigned a score of 2 . The resulting MTS risk score has a possible range of $0-5$ and is further detailed in Table 2.

As a summary measure of the overall ability of the MTS risk score to predict MTS, the estimated area under the receiver operating characteristic curve was 0.96 (95\% CI: 0.93-1.00), indicating a very high level of discrimination between MTS and non-MTS patients. The proportion of patients with MTS according to MTS risk score is shown in Table 3, and estimates

Table 4 Sensitivity, specificity, positive predictive value, and negative predictive value according to the Mayo MTS risk score

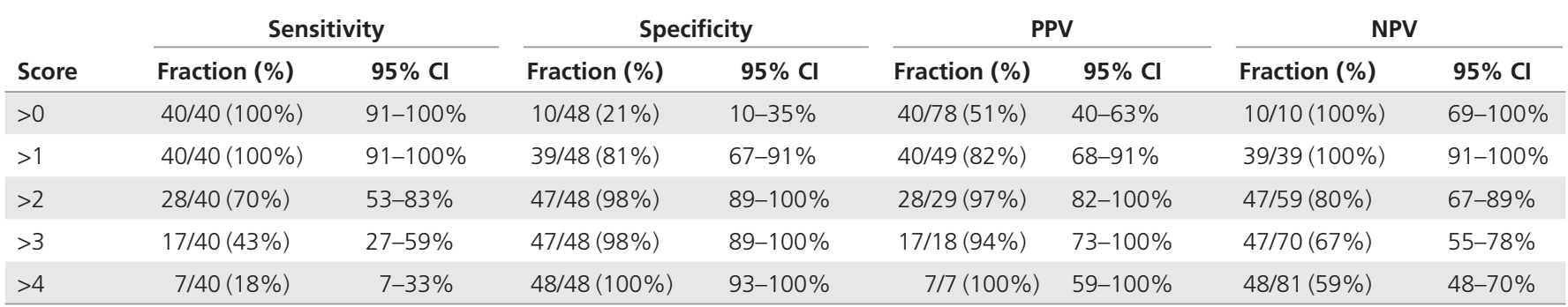

The area under the receiver operating characteristic curve for the MTS risk score in the detection of MTS was 0.96 (95\% CI: 0.93-1.00).

$\mathrm{Cl}$, confidence interval; MTS, Muir-Torre syndrome; NPV, negative predictive value; PPV, positive predictive value. 
of sensitivity, specificity, positive predictive value, and negative predictive value are shown in Table 4. As can be seen in these two tables, based on our proposed MTS risk score, a score of 2 or higher may show the most promise in differentiating between MTS and non-MTS patients, as evidenced by both high sensitivity (100\%) and high specificity (81\%). None of the 40 patients with a risk score less than 2 had MTS. We did consider that the sensitivity of our MTS risk score was attributed to the use of different scores for its individual components. The inclusion of personal history of colon cancer, family history of colon cancer, and Amsterdam II criteria, and noted no improvement in sensitivity. The MTS risk score could not be calculated for one patient since their total number of SNs was unavailable.

\section{DISCUSSION}

We identified several factors associated with a final diagnosis of MTS and created a scoring algorithm to better identify those at risk of MTS. Although promising, given the high estimates of diagnostic utility, the Mayo MTS risk score requires validation in an independent group of patients.

SNs may serve as a "herald" lesion for colon cancer in MTS patients. Therefore, all patients presenting with a SN should be screened (regarding personal and family history) for clues suggestive of MTS so that subsequently identified patients and at-risk family members may enter into cancer screening and prevention programs. Three patients had a diagnosis of a $\mathrm{SN}$ that preceded their colon cancer diagnosis. If a diagnosis of MTS had been made when the patients initially presented with a SN, yearly colonoscopy could have been recommended, which may very well have prevented their colon cancers.

Based on the data presented, we recommend that an evaluation for LS be considered in all patients who have a Mayo MTS risk score of 2 or higher. Our study provides further evidence that MMR IHC evaluation on SNs is prone to false-positive results, with an estimated false-positive rate of $56 \%$. We acknowledge that this estimated false-positive rate could slightly decrease as testing technologies improve and the underlying molecular mechanisms leading to the development of SNs are discovered. With this high false-positive rate, we recommend that the first step to further evaluating patients presenting with SNs who have a MTS risk score of 2 or higher be MMR IHC on the patient's colon and/or endometrial cancers when possible. This recommendation is based on the fact that the specificity of MMR IHC on these tumor types is much higher than that of MMR IHC on SNs. If MMR IHC on colon or endometrial cancer tissue is not possible, we would then recommend germline MMR genetic testing with additional consideration of $M U T Y H$ genetic testing in certain clinical scenarios (SNs and colon cancer can also be seen in carriers of MUTYH mutations). ${ }^{1}$ Next-generation sequencing panels are now available that include all of the MMR genes and also the MUTYH gene.

If MMR IHC results of a $\mathrm{SN}$ are available, they should not always be used to guide which genetic test is ordered. This study and our previous publication report a few patients for whom MMR IHC results indicated a mutation in a particular MMR gene, when in fact the mutation was in a different MMR gene. ${ }^{1}$

hMSH2 mutation carriers are known to have higher rates of urinary tract cancers (specifically renal pelvis and ureter) and certain other extracolonic malignancies compared to LS patients with mutations in other MMR genes. ${ }^{6}$ In this cohort, $h M S H 2$ mutation carriers also appear to be at increased risk for SNs. This relationship may be the result of a specific genotype-phenotype correlation, with carriers of the IVS5+3A $>$ T mutation as well as other mutations involving exons 5 and 6 overrepresented among hMSH2 mutation carriers.

Our study group had a high prevalence of MMR germline mutation carriers (45\%); we previously reported a prevalence of $17 \%$ in patients with an SN presenting to a dermatologist. Applying the Mayo MTS risk score to a population with a lower prevalence (17\%, rather than $45 \%)$, sensitivity, specificity, and negative predictive value should be unchanged; however, positive predictive value would decrease from $82 \%$ to an estimated $52 \%$.

Patients with SNs are typically only referred to genetics for further workup if the MMR IHC staining pattern shows a loss of expression of one or more MMR proteins. Our sample included only two patients whose MMR IHC results were normal (retention of all four proteins) for all SNs tested. It is not known whether our proposed Mayo MTS risk score would be valid in patients with normal MMR IHC on their SNs. It is also important to note the possibility of a type II error (i.e., false-negative findings) considering the small sample size. Another limitation is that germline MMR genetic testing was sometimes guided by MMR IHC pattern. Only 6 of 89 patients (7\%) included in this study had complete sequencing and deletion/duplication analysis for all known MMR genes ( $h M L H 1, h M S H 2, h M S H 6$ PMS2, and EPCAM). This raises the possibility that a few germline mutations may have been missed based on a lack of complete gene sequencing and deletion/duplication analysis of all MMR genes. A summary of the results of genetic testing can be found in Supplementary Table S6 online. Of course, there is always the possibility that a few patients may have germline mutations present in the MMR genes that are not detectable by current methods and clinical assays.

Finally, we encourage all medical providers attending to LS patients to educate their patients on the importance of self skin checks for lesions suspicious for SNs and to include a skin exam as part of their yearly comprehensive medical evaluations.

\section{SUPPLEMENTARY MATERIAL}

Supplementary material is linked to the online version of the paper at http://www.nature.com/gim

\section{ACKNOWLEDGMENTS}

A Mayo Clinic transitional grant supported this work. 


\section{DISCLOSURE}

The authors declare no conflict of interest.

\section{REFERENCES}

1. Roberts ME, Riegert-Johnson DL, Thomas BC, et al. Screening for Muir-Torre syndrome using mismatch repair protein immunohistochemistry of sebaceous neoplasms. J Genet Couns 2013;22:393-405.

2. Hampel H, Frankel WL, Martin E, et al. Feasibility of screening for Lynch syndrome among patients with colorectal cancer. J Clin Oncol 2008;26: 5783-5788.

3. Hampel H, Stephens JA, Pukkala E, et al. Cancer risk in hereditary nonpolyposis colorectal cancer syndrome: later age of onset. Gastroenterology 2005; 129:415-421.
4. Lindor NM, Burgart LJ, Leontovich $\mathrm{O}$, et al. Immunohistochemistry versus microsatellite instability testing in phenotyping colorectal tumors. J Clin Oncol 2002:20:1043-1048.

5. Zhang L. Immunohistochemistry versus microsatellite instability testing for screening colorectal cancer patients at risk for hereditary nonpolyposis colorectal cancer syndrome. Part II. The utility of microsatellite instability testing. J Mol Diagn 2008;10:301-307.

6. van der Post RS, Kiemeney LA, Ligtenberg MJ, et al. Risk of urothelial bladder cancer in Lynch syndrome is increased, in particular among MSH2 mutation carriers. J Med Genet 2010;47: 464-470.

7. Desai DC, Lockman JC, Chadwick RB, et al. Recurrent germline mutation in MSH2 arises frequently de novo. J Med Genet 2000; 37(9): 646-652. 